\title{
Body dysmorphic disorder in female Swedish dermatology patients
}

Sabina Brohede, Yvonne Wyon, Gun Wingren, Barbro Wijma and Klaas Wijma

The self-archived postprint version of this journal article is available at Linköping University Institutional Repository (DiVA):

http:/ / urn.kb.se/ resolve?urn=urn:nbn:se:liu:diva-143231

N.B.: When citing this work, cite the original publication.

Brohede, S., Wyon, Y., Wingren, G., Wijma, B., Wijma, K., (2017), Body dysmorphic disorder in female Swedish dermatology patients, International J ournal of Dermatology, 56(12), 1387-1394.

https:// doi.org/ 10.1111/ijd.13739

Original publication available at:

https:// doi.org/ 10.1111/ijd.13739

Copyright: Wiley (12 months)

http:// eu.wiley.com/WileyCDA/ 


\title{
Body dysmorphic disorder in female Swedish dermatology patients
}

\author{
Brohede S, M.D., Ph.D. ${ }^{a b *}$, Wyon Y, M.D., Ph.D. ${ }^{c}$, Wingren G, Ph.D. ${ }^{d}$, \\ Wijma B, M.D., Profe, and Wijma K, Prof ${ }^{a}$
}

a Unit of Medical Psychology, Department of Clinical and Experimental Medicine, Faculty of Medicine and Health Sciences, Linköping University, Linköping, Sweden.

b Department of Paediatrics and Department of Clinical and Experimental Medicine, Linköping University, Linköping, Sweden.

c Department of Dermatology and Venereology and Department of Clinical and Experimental Medicine, Linköping University, Linköping, Sweden.

d Occupational and Environmental Medicine, Department of Clinical and Experimental Medicine, Faculty of Medicine and Health Sciences, Linköping University, Linköping, Sweden.

e Gender and Medicine, Department of Clinical and Experimental Medicine, Faculty of Medicine and Health Sciences, Linköping University, Linköping, Sweden.

*Corresponding author:

Sabina Brohede, M.D., Ph.D.

Department of Clinical and Experimental Medicine

Entrance $75 / 78$, House $511,12^{\text {th }}$ floor

Campus US

S-581 83 Linköping

Sweden

Tel: +46702991428

E-mail: Sabina.Brohede@liu.se

Word count (Introduction through Discussion excluding references) $=3000$ Number of tables: 3

Number of figures: 2

Conflict of interest: All authors declare that there are no conflicts of interest. 
BDD in female dermatology patients

\section{ABSTRACT}

Background: Individuals with body dysmorphic disorder (BDD) are highly distressed and impaired owing to perceived defects in their physical appearance that are not noticeable to others. They are frequently concerned about their skin and often present to dermatologists rather than psychiatrists. However, BDD patients attending dermatology clinics may be at risk of not receiving an appropriate assessment and beneficial treatment. The aims of the present study were to estimate the BDD prevalence rate among Swedish female dermatology patients and to assess the psychological condition of BDD patients compared to that of other dermatology patients. Methods: The occurrence of BDD was estimated using the Body Dysmorphic Disorder Questionnaire (BDDQ), a validated self-report measure for BDD. Symptoms of depression and anxiety were measured by the Hospital Anxiety and Depression Scale (HADS), and quality of life was assessed using the Dermatology Life Quality Index (DLQI). Results: The prevalence rate of BDD among female Swedish dermatology patients was 4.9\% (95\% CI 3.2-7.4). Anxiety (HADS A $\geq 11$ ) was four-fold more commonly reported by patients with positive BDD screening (48\% vs. 11\%), and depression (HADS D $\geq 11$ ) was over ten-fold more common in patients with positive BDD screening (19\% vs. 1.8\%) $(\mathrm{p}<0.001)$. The median DLQI score was 18 in the BDD group, compared to a score of 4 in the non-BDD group $(\mathrm{p}<0.001)$. Conclusions: Our results indicate that BDD is fairly common among female Swedish dermatology patients (4.9\%) and that BDD patients have high levels of depression and anxiety and severely impaired quality of life. 
BDD in female dermatology patients

\section{INTRODUCTION}

Approximately one-third of dermatology patients are estimated to have underlying psychiatric comorbidity, and psychiatric illness may either be the cause or the consequence of dermatologic disease.1,2 Individuals with body dysmorphic disorder (BDD) are highly distressed and impaired owing to defects they perceive in their physical appearance that are not observable to others; if a minor physical anomaly is present, the individual's concern is markedly excessive. ${ }^{3}$ Most individuals have concerns involving the face or head, usually the skin (e.g., perceived acne, scars, wrinkles or paleness), hair (e.g., thinning hair or excessive body or facial hair), and nose. ${ }^{4,5}$ BDD sufferers engage in excessive grooming, skin picking, mirror checking, and camouflaging of their appearance, with the aim of correcting, hiding, or distracting others from perceived defective parts of the body. Focusing on unattractive parts of the body, rumination, mental rituals or other mental acts are also often reported 6,7 These preoccupations are generally time-consuming, occurring on average 3-8 hours a day, and are difficult to resist or control. ${ }^{8}$ The condition causes impaired functioning in relationships, socializing, and intimacy, as well as a decreased ability to function at work, in school, or in other daily activities. ${ }^{4,9}$ Anxiety disorders, depression, and eating disorders frequently co-occur with BDD and the disorder is associated with significant suicidality. ${ }^{10-12}$

The onset of BDD commonly occurs during adolescence; however, patients are generally diagnosed 10-15 years later.11,13 BDD patients generally feel misunderstood and are secretive about their symptoms because they think they will be viewed as vain or narcissistic. Moreover, most BDD patients have poor insight into their illness; 30 to $60 \%$ of patients are even delusional regarding their perceived appearance flaws and they do 
BDD in female dermatology patients

not recognize a need for psychological or psychiatric treatment. ${ }^{14}$ As the skin is one of the most commonly reported areas of concern in BDD, many BDD sufferers seek dermatological or surgical treatment in an attempt to relieve their symptoms. ${ }^{15,16}$ However, several studies indicate that what these specialists can offer by means of appearance-enhancing treatments, such as various dermatological treatments or cosmetic surgery, usually do not result in any decrease in BDD symptom severity. Following cosmetic treatments, some individuals instead develop new appearance concerns, and, unfortunately, even symptom exacerbations are not uncommon. ${ }^{15,16}$ Therefore, dermatologists play an important role in suspecting BDD in their differential diagnosis consideration and referring these patients for appropriate assessment and therapy. Treatments that have been shown to be effective for BDD are high-dose serotonin-reuptake inhibitors and cognitive behavioural therapy. ${ }^{8,16-19}$ However, BDD often goes unrecognized by dermatologists, plastic surgeons, and other physicians whom these patients approach first in their attempts to correct their perceived flaws. ${ }^{12,20}$

BDD prevalence rates of 1.7 to $2.4 \%$ have been identified in general population samples in Germany, Sweden, and the United States. ${ }^{21-24}$ In dermatology settings, a few studies have systematically assessed the occurrence of BDD, and have found higher prevalence rates of 4.5 to $14 \% . .^{25-32}$ Some studies have reported lower prevalence rates among general dermatology patients (2.1 to 6.7\%) than among cosmetic dermatology patients $(7.5$ to $14 \%) \cdot{ }^{29-31}$

In summary, BDD patients attending dermatology clinics may be at risk of not receiving an appropriate assessment and beneficial treatment. Because there are no known data 
BDD in female dermatology patients

on BDD occurrence in clinical settings in Sweden, the primary aim of this study was to use a validated questionnaire to estimate the BDD prevalence rate among Swedish female dermatology patients. Women were chosen because the questionnaire had previously been validated in Swedish women. To estimate the psychological condition of BDD patients compared to that of other dermatology patients, the secondary aims were to study the co-occurrence of depression and anxiety, assess quality of life, and investigate BDD patients' reasons for seeking dermatologic care. 
BDD in female dermatology patients

\section{METHOD}

\section{Participants}

From February to September 2013, 523 patients were consecutively enrolled at two tertiary care dermatologic outpatient clinics in Sweden. The eligibility criteria included female non-cancer, general dermatology patients aged 18-60 years. Validated selfscreening instruments were used to assess the prevalence of BDD, symptoms of depression and anxiety, and quality of life. A total of 425 women participated in the study after excluding seven responders because of non-response on items required for BDD diagnosis. Thus, the response rate was $81 \%$. The study was approved by the Regional Ethics Board, Linköping, Sweden.

\section{Measurements}

The Body Dysmorphic Disorder Questionnaire (BDDQ) is a brief, self-report measure that is derived from the DSM-IV diagnostic criteria for BDD (Fig. 1). The questionnaire was developed as a screening instrument for BDD and has exhibited high sensitivity (100\%) and specificity (89 to 93\%) when validated in psychiatric samples. ${ }^{33,34}$ The BDDQ has also been validated against the Structured Clinical Interview for DSM-IV (SCID) in a sub-sample $(\mathrm{n}=127)$ of a facial cosmetic surgery sample, and displayed a sensitivity of $100 \%$ and a specificity of $91 \% .35$ The BDDQ dermatology version, a modification of the BDDQ with continuous scoring on items evaluating distress and impairment, was validated in dermatology patients seeking cosmetic surgical consultation, and presented high sensitivity and specificity $(100 \%$ and $92 \%$ respectively). ${ }^{36}$ The Swedish translation of the BDDQ has been validated against face-toface diagnostic interviews using the SCID in a community sample of Swedish women and exhibited a sensitivity of $94 \%$ and a specificity of $90 \% .{ }^{37}$ The original intention was to 
BDD in female dermatology patients

complement a positive BDDQ screening with a diagnostic interview using the SCID and a dermatological evaluation of the perceived appearance flaws. All patients with positive screening on the BDDQ were contacted via telephone and mail and invited to a diagnostic interview. Of the 21 patients who screened positive for BDD, eight women declined participation and six women could not be reached. Since only one-third of those screening positive for BDD participated in the diagnostic interview, the usefulness of the intended method for BDD assessment was considerably limited. Therefore, the BDD prevalence rate in this study is reported based on positive BDDQ screening.

The Hospital Anxiety and Depression Scale (HADS) is a validated 14-item self-report screening scale that has performed well in screening for anxiety disorders and depression in patients from non-psychiatric hospital, general practice, and psychiatric clinics, as well as in individuals in the general population. ${ }^{38,39}$ The scale assesses the occurrence of symptoms of depression and anxiety during the previous week on a seven-item anxiety subscale and a seven-item depression subscale; each item is scored from 0 to 3 (maximum score of 21 in each subscale). Snaith ${ }^{40}$ proposed that a score of 11 or higher indicates the probable presence of a mood disorder. Most studies that have used a HADS cut-off score of 11 to denote clinically significant depression/anxiety have exhibited specificities of over $90 \% \cdot{ }^{39}$ Cronbach's alpha was 0.84 for HADS anxiety and 0.83 for HADS depression in the present sample.

The Dermatology Life Quality Index (DLQI) is a widely used and validated self-report tool specific for dermatology. ${ }^{41,42}$ The DLQI is comprised of 10 questions assessing quality of life in six subdomains: symptoms and feelings, daily activities, leisure, work and school, personal relationships, and treatment. Respondents indicate the extent to 
BDD in female dermatology patients

which they have experienced certain problems during the previous week. Response options are on a four-point Likert scale from 'not at all/not relevant' to 'very much.' Scores for each item range from 0 to 3 and are summed to create an overall DLQI score; the higher the score, the more the quality of life is impaired. The total DLQI score is interpreted in an ordinal scale as follows: $0-1=$ no effect at all on the patient's life, $2-5=$ small effect, 6-10 = moderate effect, 11-20 = very large effect, and 21-30 = extremely large effect on the patient's life. The subdomains are comprised of 1 or 2 questions and, thus, have a maximum score of 3 or 6 . Individual subdomain scores are expressed as a percentage (0-100\%) of the maximum subdomain score. Cronbach's alpha for the DLQI was 0.91 in the present sample.

\section{Statistical methods}

The reliability of the HADS and DLQI scales was estimated by calculating Cronbach's alpha. Sociodemographics and HADS and DLQI scores were compared between patients with a positive and negative BDD screening (BDD/no BDD). Between-group differences for sociodemographics were assessed using the independent samples t-test for continuous variables (age), and binary logistic regression for categorical variables. Anxiety and depression were estimated from the HADS scores as dichotomous variables (HADS $\geq 11$ ), and binary logistic regression was used to analyse between-group differences. The Mann-Whitney U test was used to compare the total DLQI scores and the individual DLQI subdomain scores, and ordinal logistic regression was used for the ordinal interpretation of the DLQI scores. We present the difference in the median total DLQI scores and the difference in the mean scores for the individual subdomains, as these scores are expressed as percentages of the maximum subdomain scores. From the logistic regression models we report odds ratios (ORs) as effect sizes. The adjusted ORs 
BDD in female dermatology patients

were assessed after adjusting for age. Effect sizes for the differences in DLQI scores were estimated by the product-moment correlation coefficient $r$, calculated from the Z-score obtained by the Mann-Whitney U tests, ${ }^{43}$ and were interpreted as outlined by Cohen, ${ }^{44}$ $0.1=$ small effect, $0.3=$ medium effect, and $0.5=$ large effect. All analyses were performed using IBM SPSS Statistics version 22.0 software. The level of statistical significance was set at $\mathrm{p}<0.05$. 


\section{RESULTS}

\section{Prevalence of body dysmorphic disorder}

*Figure 1 approximately here*

The results of the BDDQ are presented in Fig. 1. Almost half of the respondents (46\%) reported being very concerned about some parts of their body that they considered especially unattractive, and 33\% reported being preoccupied by these concerns. Approximately half of the patients with a preoccupation with appearance concerns had primary concerns of not being thin enough or feared becoming fat and were excluded from further BDD assessment. In total, 4.9\% (95\% CI 3.2-7.4) of the patients screened positive for BDD.

The patients with positive BDD screening were younger, with a mean age of 31 years, compared to 40 years in patients without BDD ( $\mathrm{p}=0.001)$ (Table 1). Following adjustments for age, the patients with positive BDD screening were more commonly unemployed (OR 5.2, 95\% CI 1.3-20.3) or on sick leave (OR 4.1, 95\% CI 1.0-16.5) compared to the patients without BDD.

*Table 1 approximately here*

The body areas that the patients who screened positive for BDD reported that they were preoccupied with, together with the patients' reasons for attending the clinic, are presented in Table 2. For some patients, their reasons for attending the clinic were presumably to seek treatment for their perceived appearance flaw (e.g., seeking treatment for acne when preoccupied with the facial skin). However, not all patients sought dermatologic care because of their reported appearance preoccupations, and, for 
BDD in female dermatology patients

some patients, it was not obvious whether their preoccupation was the reason for attending the clinic.

*Table 2 approximately here*

Depression, anxiety, and quality of life

Depression (HADS D $\geq 11$ ) was reported by $2.7 \%$ and anxiety (HADS A $\geq 11$ ) was reported by $13.2 \%$ of the total sample. Depression was over ten-fold more common in patients with positive BDD screening (19\% vs. 1.8\%) ( $\mathrm{p}<0.001)$, and anxiety was four-fold more common in patients with positive BDD screening (48\% vs. $11 \%)(\mathrm{p}<0.001)$ (Table 3). The median total DLQI score was 18 in the BDD group, compared to 4 in the non-BDD group ( $\mathrm{p}<0.001$ ) (Table 3). The interpretation of the DLQI scores with regard to the effect on the patients' lives is illustrated in Fig. 2, indicating that quality of life was significantly more affected in patients with positive BDD screening (adjusted OR 10.5 (95\% CI 4.5-4.8), $\mathrm{p}<0.001)$. All DLQI subdomains were significantly more affected in patients with positive BDD screening.

*Table 3 approximately here*

*Figure 2 approximately here* 
BDD in female dermatology patients

\section{DISCUSSION}

We identified a BDD prevalence of 4.9\% (95\% CI 3.2-7.4) among female Swedish dermatology patients $(n=425)$, as estimated using the BDDQ. These results confirm earlier research reporting that BDD is fairly common in dermatology settings and indicate that BDD is more than twice as common in dermatology patients than in the general population of Swedish women $(2.1 \%) .{ }^{23}$ The BDD prevalence rate found in our sample was lower than that in some previous studies of general dermatology samples. About $14 \%$ of American general dermatology patients and 7.9\% of German dermatology outpatients have screened positive for BDD by the use of questionnaires. ${ }^{25,26,32}$ Studies that have used the SCID to diagnose BDD have found prevalence rates of $6.7 \%$ and $8.8 \%$ among Brazilian and Turkish general dermatology patients. ${ }^{27,29}$ Two recent studies that used screening questionnaires, reported a lower BDD prevalence rate of $4.2 \%$ and $2.1 \%$ among Turkish and Indian general dermatology patients. ${ }^{30,31}$ In our study, we used an instrument with good validity, that excludes patients with primary weight concerns to avoid over-diagnosing BDD when an eating disorder may be a more accurate diagnosis. ${ }^{45}$ However, eating disorders and BDD can be comorbid conditions, in which case both disorders should be diagnosed. ${ }^{46}$ Therefore, screening for BDD using the BDDQ instead involves a risk of under-diagnosis of BDD. The varying rates of BDD obtained may be due to differences in methods of assessment and sample sizes as well as cultural differences and different health care systems. BDD patients may attend dermatology clinics to a lesser degree in Sweden because some appearance-enhancing treatments (e.g., fillers, minor surgery, threads, Botox etc.) are not performed in Swedish dermatology clinics. In private dermatology settings, a higher BDD prevalence may therefore have been found. A limitation of the present study is that the BDD prevalence was estimated by a screening questionnaire instead of using a face-to-face diagnostic 
method. The unwillingness by the patients with positive BDD screening to participate in a face-to-face assessment may be indicative of the nature of BDD. However, dermatology patients may theoretically screen falsely positive for BDD on the BDDQ owing to psychiatric symptoms secondary to disfiguring dermatological conditions, ${ }^{2}$ as the appearance defects could not be objectively assessed. Nevertheless, in the study by Conrado et. al. ${ }^{29} 32$ of 36 patients who screened positive for BDD by the BDDQ were also diagnosed with BDD using the SCID, suggesting that the BDDQ performed well as a diagnostic instrument in dermatology patients.

Among the patients with positive BDD screening, the reason for seeking dermatologic treatment was not always their main body area of concern. This result is consistent with findings in a previous study in which, for more than half of the BDD patients, their dermatologic symptom was not the same as their major BDD concern. ${ }^{29}$ These authors hypothesized that patients with BDD seeking dermatologic care may not be able to precisely report their symptom or real complaint. Another explanation may be that, as many BDD patients scrutinize their skin and facial features, in addition to their perceived defects, they may become aware of other skin changes and seek dermatological care. The shame associated with the disorder may also prevent BDD patients from revealing their "real" BDD preoccupations to health care professionals. ${ }^{20}$

To the best of our knowledge, no previous studies have reported data on the psychological condition of BDD patients in dermatology settings. The high levels of depression and anxiety in patients who screened positive for BDD in our study support the findings of previous studies of BDD patients. In the largest samples of BDD patients, the lifetime prevalence of major depression, as assessed by the SCID, was 75 to $76 \%$ and 
the current prevalence was $58 \% .^{10,11}$ In those studies, the lifetime prevalence of anxiety disorders (most commonly social anxiety disorder) was 64 to $73 \%$ and the current prevalence was 55\%. Studies using the screening questionnaire Montgomery and Åsberg Depression Scale (MADRS) have reported depression in 28 to 50\% of BDD patients (defined as a score of $>20$ to denote clinically significant depression). ${ }^{47,48}$ The rates of depression (19\%) and anxiety (48\%) found in BDD patients in the present study were thus lower than those in previous studies, although the rates are difficult to compare owing to differences in assessment methods. In the total sample, we found depression in $2.7 \%$ and anxiety in $13.2 \%$ of the dermatology patients, which were lower rates compared with results from a large European multicentre study of dermatological out-patients $(n=3635)$ that reported depression in $10 \%$ and anxiety in $17 \%$ of the patients based on a HADS cut-off score of $11 .{ }^{49}$ Although other Scandinavian countries were included, Sweden was not represented in the multicentre study, and existing data from Sweden are limited. Therefore, more studies are needed to compare the psychological condition of BDD patients with that of other dermatology patients.

Quality of life was severely impaired in the patients with positive BDD screening, with a median DLQI score of 18, which is compatible with very large effects on the patients' lives. There are no norm data for the DLQI; however, a mean total DLQI score of 0.5 for healthy controls and a mean score of 7.3 for dermatology patients has been reported. ${ }^{41}$ As a comparison with scores for some of the dermatology disorders associated with considerably impaired quality of life, recent reviews have reported mean DLQI scores ranging from 1.7 to 18.2 for psoriasis, ${ }^{50} 2.0$ to 17.7 for acne,,$^{51} 4.3$ to 17.3 for rosacea, ${ }^{51}$ and 1.8 to 15 for vitiligo. ${ }^{52}$ In our study, the patients with positive BDD screening were 
BDD in female dermatology patients

also unemployed and on sick leave to a higher degree, findings that indicate potential consequences of the disorder.

In conclusion, this is the largest study assessing BDD prevalence in dermatology settings, and the results confirm that BDD seems to be fairly common among patients attending dermatology clinics. The patients who screened positive for BDD experienced symptoms of anxiety and depression to a high degree, and their quality of life was severely impaired. As outlined by Gupta and Gupta,53 it is important to rule out body image pathologies before initiating dermatologic therapies because patients with BDD are often dissatisfied with treatment outcomes and because body image dissatisfaction is associated with increased morbidity, intentional self-injury, and suicide. Thus, even if visible dermatological symptoms are mild, it is important to assess the emotional consequences of the patients' symptoms. Phillips and Dufresne ${ }^{54}$ recommended that BDD patients should be referred to mental health professionals for treatment and emphasized the need to provide BDD patients with psycho-education about the disorder, rather than dismissing their concerns as trivial, trying to reassure them that treatments are unnecessary, or trying various appearance-enhancing treatments. Because dermatologists may be the first or only health care professionals approached by BDD patients, an increased awareness of BDD among dermatologists is vital to ensure that these patients receive the appropriate care.

\section{ACKNOWLEDGEMENTS}

Dermatologist Marianne Maroti, M.D., contributed to the planning and implementation of the study. 
BDD in female dermatology patients

\section{REFERENCES}

1. Brown GE, Malakouti M, Sorenson E, et al. Psychodermatology. Adv Psychosom Med 2015;34:123-34.

2. Yadav S, Narang T, Kumaran MS. Psychodermatology: a comprehensive review. Indian J Dermatol Venereol Leprol 2013;79(2):176-92.

3. American Psychiatric Association. Diagnostic and statistical manual of mental disorders. $5^{\text {th }}$ ed. Arlington, VA: American Psychiatric Association, 2013.

4. Phillips KA. Understanding body dysmorphic disorder : an essential guide. Oxford: Oxford University Press, 2009.

5. Phillips KA, Diaz SF. Gender differences in body dysmorphic disorder. J Nerv Ment Dis 1997;185(9):570-7.

6. Phillips KA. Body Dysmorphic Disorder: Common, Severe and in Need of Treatment Research. Psychother Psychosom 2014;83(6):325-9.

7. Kollei I, Martin A. Body-related cognitions, affect and post-event processing in body dysmorphic disorder. J Behav Ther Exp Psychiatry 2014;45, 144-51.

8. Phillips KA, Hollander E. Treating body dysmorphic disorder with medication: evidence, misconceptions, and a suggested approach. Body image 2008;5(1):1327.

9. Perugi G, Giannotti D, Frare F et al. Prevalence, phenomenology and comorbidity of body dysmorphic disorder (dysmorphophobia) in a clinical population. Int J Psychiatry Clin Pract 1997;1(2):77-82.

10. Gunstad J, Phillips KA. Axis I comorbidity in body dysmorphic disorder. Compr psychiatry 2003;44(4):270-6. 
11. Phillips KA, Menard W, Fay C, Weisberg R. Demographic characteristics, phenomenology, comorbidity, and family history in 200 individuals with body dysmorphic disorder. Psychosomatics 2005;46(4):317-25.

12. Phillips KA. Suicidality in Body Dysmorphic Disorder. Prim psychiatry 2007;14(12):58-66.

13. Veale D. Body dysmorphic disorder. Postgrad Med J 2004;80(940):67-71.

14. Phillips KA, Stein DJ, Rauch SL, et al. Should an obsessive-compulsive spectrum grouping of disorders be included in DSM-V? Depress Anxiety 2010;27(6):52855.

15. Phillips KA, Grant J, Siniscalchi J, Albertini RS. Surgical and nonpsychiatric medical treatment of patients with body dysmorphic disorder. Psychosomatics 2001;42(6):504-10.

16. Crerand CE, Phillips KA, Menard W, Fay C. Nonpsychiatric medical treatment of body dysmorphic disorder. Psychosomatics 2005;46(6):549-55.

17. Enander J, Andersson E, Mataix-Cols D, et al. Therapist guided internet based cognitive behavioural therapy for body dysmorphic disorder: single blind randomised controlled trial. BMJ 2016;352.

18. Veale D, Anson M, Miles S, et al. Efficacy of cognitive behaviour therapy versus anxiety management for body dysmorphic disorder: a randomised controlled trial. Psychother Psychosom 2014;83(6):341-53.

19. Wilhelm S, Phillips KA, Didie E, et al. Modular cognitive-behavioral therapy for body dysmorphic disorder: a randomized controlled trial. Behav Ther 2014;45(3):314-27. 
20. Brohede S, Wijma B, Wijma K, Blomberg K. 'I will be at death's door and realize that I've wasted maybe half of my life on one body part': the experience of living with body dysmorphic disorder. Int J Psychiatry Clin Pract 2016;20(3):191-8.

21. Koran LM, Abujaoude E, Large MD, Serpe RT. The prevalence of body dysmorphic disorder in the United States adult population. CNS Spectr 2008;13(4):316-22.

22. Rief W, Buhlmann U, Wilhelm S, et al. The prevalence of body dysmorphic disorder: a population-based survey. Psychol med 2006;36(6):877-85.

23. Brohede S, Wingren G, Wijma B, Wijma K. Prevalence of body dysmorphic disorder among Swedish women: A population-based study. Compr psychiatry 2015;58:108-15.

24. Buhlmann U, Glaesmer H, Mewes R, et al. Updates on the prevalence of body dysmorphic disorder: a population-based survey. Psychiatry Res 2010;178(1):171-5.

25. Phillips KA, Dufresne RG, Jr., Wilkel CS, Vittorio CC Rate of body dysmorphic disorder in dermatology patients. J Am Acad Dermatol 2000;42(3):436-41.

26. Bowe WP, Leyden JJ, Crerand CE, et al. Body dysmorphic disorder symptoms among patients with acne vulgaris. J Am Acad Dermatol 2007;57(2):222-30.

27. Uzun 0, Basoglu C, Akar A, et al. Body dysmorphic disorder in patients with acne. Compr psychiatry 2003;44(5):415-9.

28. Mufaddel A, Osman OT, Almugaddam F, Jafferany M. A review of body dysmorphic disorder and its presentation in different clinical settings. Prim Care Companion CNS Disord 2013;15(4). pii: PCC.12r01464.

29. Conrado LA, Hounie AG, Diniz JB, et al. Body dysmorphic disorder among dermatologic patients: Prevalence and clinical features. JAA. 2010;63(2):235-43. 
30. Dogruk Kacar S, Ozuguz P, Bagcioglu E, et al. The frequency of body dysmorphic disorder in dermatology and cosmetic dermatology clinics: a study from Turkey. Clin Exp Dermatol 2014;39(4):433-8.

31. Thanveer F, Khunger N. Screening for Body Dysmorphic Disorder in a Dermatology Outpatient Setting at a Tertiary Care Centre. J Cutan Aesthet Surg 2016;9(3):188-91.

32. Ritter V, Flugh JW, Schliemann-Willers S, et al. Body dysmorphic concerns, social adaptation, and motivation for psychotherapeutic support in dermatological outpatients. J Dtsch Dermatol Ges 2016;14(9):901-8.

33. Phillips KA, Atala KD, Pope HG. Diagnostic instruments for body dysmorphic disorder. New Research Program and Abstracts, American Psychiatric Association 148th annual meeting, Miami. Washington, DC: American Psychiatric Press, 1995.

34. Grant JE, Kim SW, Crow SJ. Prevalence and clinical features of body dysmorphic disorder in adolescent and adult psychiatric inpatients. J Clin psychiatry 2001;62(7):517-22.

35. Dey JK, Ishii M, Phillis M, et al. Body dysmorphic disorder in a facial plastic and reconstructive surgery clinic: measuring prevalence, assessing comorbidities, and validating a feasible screening instrument. JAMA Facial Plast Surg 2015;17:137-43.

36. Dufresne RG, Phillips KA, Vittorio CC, Wilkel, CS. A screening questionnaire for body dysmorphic disorder in a cosmetic dermatologic surgery practice. Dermatol Surg 2001;27:457-62.

37. Brohede S, Wingren G, Wijma B, Wijma K. Validation of the Body Dysmorphic Disorder Questionnaire in a community sample of Swedish women. Psychiatry 
Res 2013;210(2):647-52.38. Zigmond AS, Snaith RP. The hospital anxiety and depression scale. Acta Psychiatr Scand 1983;67(6):361-70.

39. Bjelland I, Dahl AA, Tangen Haug T, Neckelman D. The validity of the Hospital Anxiety and Depression Scale. An updated literature review. J Psychosom res 2002;52(2):69-77.

40. Snaith RP. The Hospital Anxiety And Depression Scale. Health Qual Life Outcomes 2003;1(1):1-4.

41. Finlay AY, Khan GK. Dermatology Life Quality Index (DLQI)--a simple practical measure for routine clinical use. Clin Exp Dermatol 1994;19(3):210-6.

42. Basra MK, Fenech R, Gatt RM, et al. The Dermatology Life Quality Index 19942007: a comprehensive review of validation data and clinical results. $\mathrm{Br} J$ Dermatol 2008;159(5):997-1035.

43. Fritz CO, Morris PE, Richler JJ. Effect size estimates: current use, calculations, and interpretation. J Exp Psychol Gen 2012;141(1):2-18.

44. Cohen J. A power primer. Psychol Bull 1992;112(1):155-9.

45. Phillips K. The broken mirror : understanding and treating body dysmorphic disorder. Oxford: Oxford University Press, 1998.

46. Hartmann AS, Greenberg JL, Wilhelm S. The relationship between anorexia nervosa and body dysmorphic disorder. Clin Psychol Rev 2013;33(5):675-85.

47. Veale D, Boocock A, Gournay K, et al. Body dysmorphic disorder. A survey of fifty cases. Br J Psychiatry 1996;169(2):196-201.

48. van der Meer J, van Rood YR, van der Wee NJ, et al. Prevalence, demographic and clinical characteristics of body dysmorphic disorder among psychiatric outpatients with mood, anxiety or somatoform disorders. Nord J Psychiatry 2012;66: 232-8 
BDD in female dermatology patients

49. Dalgard FJ, Gieler U, Tomas-Aragones L, et al. The psychological burden of skin diseases: a cross-sectional multicenter study among dermatological out-patients in 13 European countries. J Clin Investig Dermatol 2015;135(4):984-91.

50. Korman NJ, Zhao Y, Pike J, Roberts J. Relationship between psoriasis severity, clinical symptoms, quality of life and work productivity among patients in the USA. Clin Exp Dermatol 2016;41(5):514-21.

51. Cresce ND, Davis SA, Huang WW, Feldman SR. The quality of life impact of acne and rosacea compared to other major medical conditions. J Drugs Dermatol 2014;13(6):692-7.

52. Amer AAA, Gao X-H. Quality of life in patients with vitiligo: an analysis of the dermatology life quality index outcome over the past two decades. Int J Dermatol 2016;55(6):608-14.

53. Gupta MA, Gupta AK. Evaluation of cutaneous body image dissatisfaction in the dermatology patient. Clin Dermatol 2013;31(1):72-9.

54. Phillips KA, Dufresne RG. Body dysmorphic disorder. A guide for dermatologists and cosmetic surgeons. Am J Clin Dermatol 2000;1(4):235-43. 
BDD in female dermatology patients

\section{Table and figure legends}

Table 1 Demographic data of patients with a positive BDD screening compared to that of patients without BDD

Table 2 Reasons for visiting the dermatology clinic and the body areas of concern reported by the 21 patients with a positive BDD screening

Table 3 Depression, anxiety, and quality of life in patients with a positive BDD screening compared to that in patients without BDD

Fig. 1. Proportions of the patients fulfilling the criteria for body dysmorphic disorder according to the Body Dysmorphic Disorder Questionnaire (BDDQ). Percentages are reported as the proportion of the total number of BDDQ respondents $(n=425)$.

Fig. 2. Dermatology Life Quality Index (DLQI) scores indicating the effect of their problems on quality of life in patients with a positive BDD screening compared to patients without BDD. 
Table 1 Demographic data of patients with a positive BDD screening compared towith that of patients without BDD

\begin{tabular}{|c|c|c|c|c|c|c|c|}
\hline & $\begin{array}{l}\text { Patients with } \\
\text { BDD, \% }(n=21)\end{array}$ & $\begin{array}{l}\text { Patients without } \\
\text { BDD, \% }(n=404)\end{array}$ & $\begin{array}{c}\text { Crude } \\
\text { OR }\end{array}$ & $95 \% \mathrm{CI}$ & $\begin{array}{l}\text { Adjusted } \\
\qquad \mathbf{O R}^{\mathbf{b}}\end{array}$ & $95 \% \mathrm{CI}$ & $\begin{array}{c}\text { P-value } \\
\text { (adjusted OR) }\end{array}$ \\
\hline Age: mean \pm SD & $30.9 \pm 10.7$ & $40.1 \pm 12.9$ & & & & & $0.001 *$ \\
\hline \multicolumn{8}{|l|}{ Education } \\
\hline \multicolumn{8}{|l|}{ (highest completed) } \\
\hline University & $38.1(n=8)$ & $46.2(n=186)$ & 1.00 & - & 1.00 & - & - \\
\hline High school & $42.9(n=9)$ & $48.1(n=194)$ & 1.08 & $0.41-2.86$ & 0.73 & $0.26-2.04$ & 0.552 \\
\hline Elementary school & $19.0(n=4)$ & $5.7(n=23)$ & 4.04 & $1.13-14.49$ & 3.18 & $0.83-12.17$ & 0.091 \\
\hline \multicolumn{8}{|l|}{ Marital status } \\
\hline Married & $19.0(n=4)$ & $45.8(n=185)$ & 1.00 & - & 1.00 & - & - \\
\hline In a relationship & $57.1(n=12)$ & $34.4(n=139)$ & 3.99 & $1.26-12.65$ & 2.09 & $0.56-7.78$ & 0.273 \\
\hline Single & $23.8(n=5)$ & $19.8(n=80)$ & 2.89 & $0.76-11.05$ & 1.34 & $0.29-6.29$ & 0.709 \\
\hline
\end{tabular}




\section{Employment status}

\begin{tabular}{|c|c|c|c|c|c|c|c|}
\hline Employed & $47.6(n=10)$ & $75.2(n=303)$ & 1.00 & - & 1.00 & - & - \\
\hline Student & $19.0(n=4)$ & $13.2(n=43)$ & 2.29 & $0.69-7.56$ & 0.93 & $0.25-3.41$ & 0.907 \\
\hline Unemployed & $19.0(n=4)$ & $3.5(n=14)$ & 8.66 & 2.41-31.05 & 5.23 & $1.34-20.29$ & $0.017^{*}$ \\
\hline Sick leave/ & $14.3(n=3)$ & $8.2(n=33)$ & 2.76 & $0.72-10.51$ & 4.06 & $1.00-16.48$ & $0.050^{*}$ \\
\hline \multicolumn{8}{|c|}{ Disability support } \\
\hline \multicolumn{8}{|c|}{ onthly household } \\
\hline \multicolumn{8}{|l|}{ come $(\mathrm{SEK})^{\mathrm{a}}$} \\
\hline$<10,000$ & $4.8(n=1)$ & $6.6(n=26)$ & 0.43 & $0.05-3.52$ & 0.27 & $0.03-2.2$ & 0.224 \\
\hline $10,000-29,999$ & $47.6(n=10)$ & $28.6(n=112)$ & 1.00 & - & 1.00 & - & - \\
\hline $30,000-49,999$ & $33.3(n=7)$ & $34.7(n=136)$ & 0.58 & $0.21-1.56$ & 0.78 & $0.28-2.2$ & 0.640 \\
\hline$>50,000$ & $14.3(n=3)$ & $29.3(n=118)$ & 0.29 & $0.08-1.06$ & 0.45 & $0.12-1.9$ & 0.254 \\
\hline
\end{tabular}


a $100 \mathrm{SEK}=8.51$ EUR (February 2013)

b Odds ratio adjusted for age.

* Significant difference $(\mathrm{p}<0.05)$. 
Table 2 Reasons for visiting the dermatology clinic and the body areas of concern reported by the 21 patients with a positive BDD screening

\begin{tabular}{|c|c|}
\hline Reason for seeking a dermatologist & Body area of concern/ "appearance flaw" \\
\hline acne & skin \\
\hline acne & nose, facial skin \\
\hline acne & skin of arms, shoulders, chest and face, thighs \\
\hline acne & facial skin \\
\hline acne & stomach, buttocks, breasts \\
\hline cryo therapy & being too skinny \\
\hline eczema of face and body & skin around the eyes, teeth \\
\hline folliculitis & legs \\
\hline follow-up malignant melanoma & facial skin, breasts \\
\hline itching and rash & body hair, sweating \\
\hline nevi (moles) and rash & buttocks, skin of arms and legs \\
\hline patch test & face and almost every body part \\
\hline patch test & eyes \\
\hline patch test & skin, nose, weight \\
\hline psoriasis & skin of the legs and arms \\
\hline psoriasis & skin of the scalp, elbows, and stomach \\
\hline psoriasis & skin \\
\hline rash & hips, thighs, stretch marks \\
\hline rash on hands and feet & skin of the arms and legs due to rash \\
\hline recurrent skin reactions & skin of the hands and feet \\
\hline skin lesion on the face & skin lesion on the face \\
\hline
\end{tabular}



Table 3 Depression, anxiety, and quality of life in patients with a positive BDD screening compared towith that in patients without BDD

\begin{tabular}{|c|c|c|c|c|}
\hline Variable & $\begin{array}{c}\text { Patients } \\
\text { with BDD } \\
(n=21)\end{array}$ & $\begin{array}{c}\text { Patients } \\
\text { without BDD } \\
(n=404)\end{array}$ & Effect sizec $^{c}$ & p value \\
\hline Anxiety (HADS A $\geq 11$ ) & $47.6 \%(n=10)$ & $11.4 \%(n=45)$ & $\mathrm{OR}=5.4(2.1-13.8)$ & $<0.001$ \\
\hline Depression (HADS $D \geq 11$ ) & $19.0 \%(n=4)$ & $1.8 \%(\mathrm{n}=7)$ & $\mathrm{OR}=11.8(2.9-47.6)$ & $<0.001$ \\
\hline DLQI total score (median) ${ }^{\mathrm{a}}$ & 18 & 4 & $r=0.27$ & $<0.001$ \\
\hline \multicolumn{5}{|l|}{ DLQI subdomain scoreb } \\
\hline Symptoms and feelings & 72.2 & 35.7 & $r=0.24$ & $<0.001$ \\
\hline Daily activities & 60.3 & 18.5 & $r=0.29$ & $<0.001$ \\
\hline Leisure & 51.6 & 16.1 & $r=0.25$ & $<0.001$ \\
\hline Work and school & 41.3 & 17.1 & $r=0.19$ & $<0.001$ \\
\hline Personal relationships & 54.8 & 14.5 & $r=0.30$ & $<0.001$ \\
\hline Treatment & 41.3 & 14.7 & $r=0.20$ & $<0.001$ \\
\hline
\end{tabular}

HADS=Hospital Anxiety and Depression Scale. DLQI=Dermatology Life Quality Index.

a Total DLQI scores range from 0-30; higher scores indicate more impaired quality of life.

b Mean scores for each of the six subdomains composing the DLQI expressed as a percentage of the maximum subdomain score (3 or 6).

c $\mathrm{OR}=$ Odds ratio (95\% confidence interval), adjusted for age.

$r=$ product-moment correlation $r$ estimated from the Mann-Whitney U test; $0.10=$ small effect, $0.30=$ medium effect, and 0.50=large effect.

Note: Of the total patients, 416 completed HADS-A, 408 completed HADS-D, and 423 completed the DLQI (all non-responders were in the non-BDD group and these individuals were excluded from the analyses). 


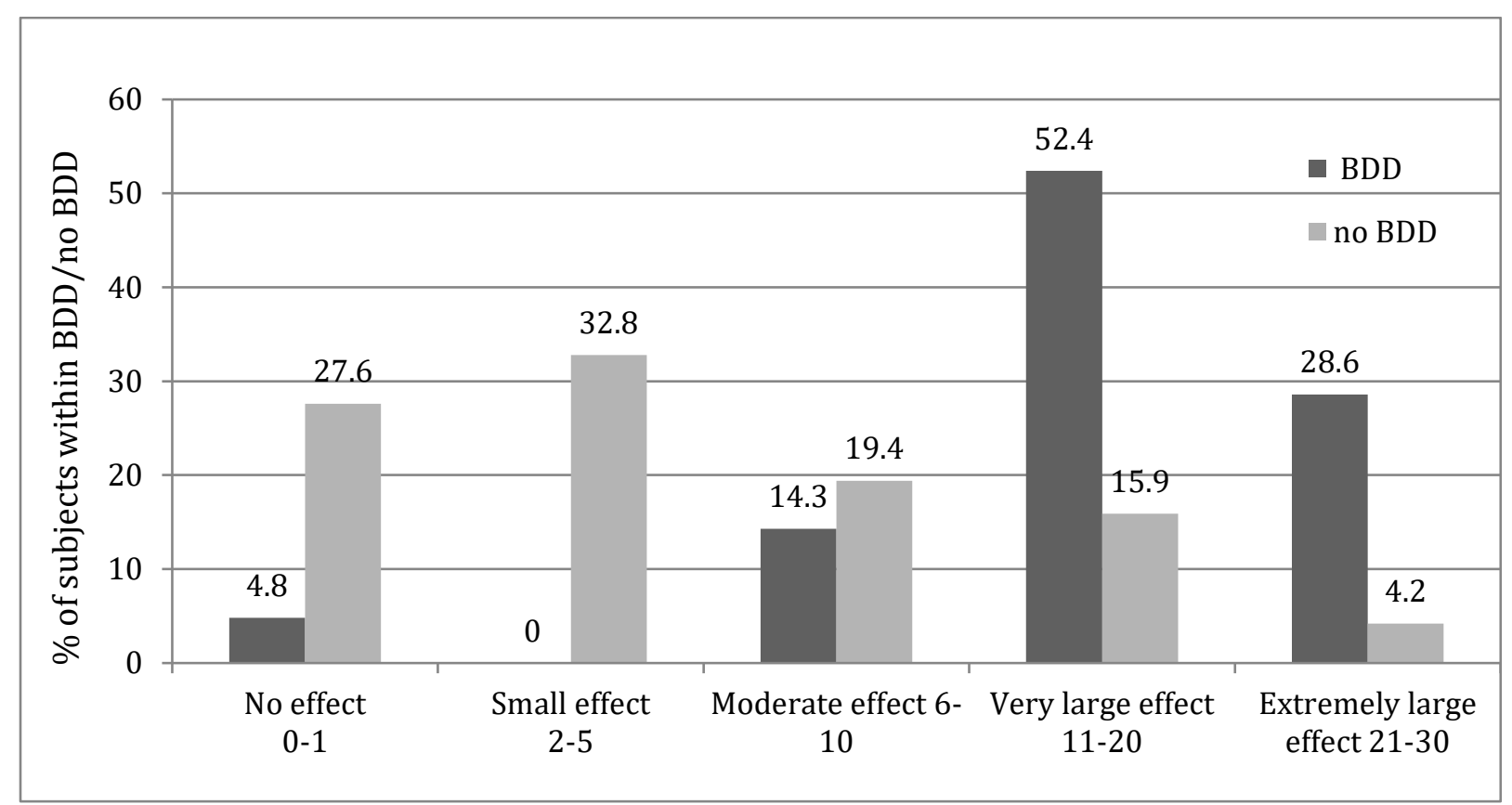

Note: DLQI scores range from 0-30; the higher the score, the more impaired the quality of life.

Figure 2. Dermatology Life Quality Index (DLQI) scores indicating the effect of their problems on quality of life in patients with a positive BDD screening compared towith patients without BDD. 